\title{
Sleeve gastrectomy relieves exocrine pancreatic insufficiency in morbidly obese patients: a prospective case-control study
} \author{
Hakan Bulus ${ }^{2}$ \\ ${ }^{1}$ Gastroenterology Department, Kecioren Training and Research Hospital, Ankara, Turkey \\ ${ }^{2}$ General Surgery Department, Kecioren Training and Research Hospital, Ankara, Turkey \\ ${ }^{3}$ Biochemistry Department, Kecioren Training and Research Hospital, Ankara, Turkey
}

Muhammet Yener Akpinar ${ }^{1}$, Dogan Ozturk², Koza Murat ${ }^{3}$, Evrim Kahramanoglu Aksoy ${ }^{1}$, Yasar Nazligul ${ }^{1}$,

Gastroenterology Rev 2019; 14 (4): 268-273

DOI: https://doi.org/10.5114/pg.2019.84223

Key words: pancreatic insufficiency, fecal elastase, sleeve gastrectomy.

Address for correspondence: Muhammet Yener Akpinar, Gastroenterology Department, Kecioren Training and Research Hospital, Sanatoryum Street, 06180 Ankara, Turkey, phone: +90 5062047839, e-mail: muhammet.yener@gmail.com

\begin{abstract}
Introduction: Bariatric surgery is the most effective treatment modality in morbidly obese patients. Compared to Roux-en $Y$ gastric bypass (RYGB), sleeve gastrectomy (SG) has better metabolic and nutritional outcomes after surgery. Exocrine pancreatic insufficiency (EPI) can be seen after RYGB but there is not any knowledge about EPI-SG association.

Aim: To assess exocrine pancreatic functions before and after the SG procedure.

Material and methods: This is a single-center, prospective and case-control study. Forty morbidly obese patients were included in the study. Their pre-operative and post-operative, third month fecal samples were collected. Exocrine pancreatic insufficiency was determined by using fecal elastase- 1 and diagnosed when fecal elastase-1 levels were $<200 \mu \mathrm{g} / \mathrm{g}$.

Results: The mean fecal elastase-1 level was $256.25 \pm 137.16 \mu \mathrm{g} / \mathrm{g}$ and the mean post-surgical fecal elastase- 1 level was $437.7 \pm 212.43 \mu \mathrm{g} / \mathrm{g}(p=0.001)$. In the pre-operative period, half of patients had FE levels under $200 \mu \mathrm{g} / \mathrm{g}$. In the third month after surgery, only 4 patients had fecal elastase-1 levels under $200 \mu \mathrm{g} / \mathrm{g}$. Comparison of fecal elastase- 1 between pre-surgery and post-surgery revealed a significant difference $(p=0.001)$.

Conclusions: This is the first study to investigate EPI-SG association. Surgery-associated morbidity and mortality are the leading limitations of bariatric surgery procedures. Exocrine pancreatic insufficiency is one of them; prior studies demonstrate its increased frequency after RYGB. Our study revealed that SG relieves exocrine pancreatic insufficiency.
\end{abstract}

\section{Introduction}

Exocrine pancreatic insufficiency (EPI) is a clinical entity characterized by deficient exocrine pancreatic enzymes that results in altered digestion [1, 2]. Various causes of EPI have been identified. Causes of EPI can be broadly categorized into pancreatic or extrapancreatic diseases. Prior studies revealed that EPI can occur in the course of diabetes mellitus (DM), obesity and celiac disease [3]. Exocrine pancreatic insufficiency was also noted after upper gastrointestinal system surgery [4]. Beside pancreatic resections, gastric resections can induce EPI in differential pathological ways [5, 6]. Also type of gastric resection (subtotal versus total) and additional intestinal anastomosis techniques can in- fluence the severity of EPI [7]. In EPI, especially lipase falls below $5-10 \%$ of normal level, steatorrhea, weight loss and symptoms associated with decreased quality of life levels occur. Suspicion and correct diagnosis are essential for EPI because enzyme replacement therapy frequently relieves the patient's symptoms and quality of life.

Obesity and related complications are a major health problem in developed and also in developing countries. Beside diet, exercise and medical treatments, bariatric surgery gives a treatment opportunity for obese patients. Especially in morbidly obese patients, bariatric surgery achieves efficient weight loss and relieves obesity-related complications. Over the years, various surgical techniques have been reported within bar- 
iatric surgery with high clinical success. Nevertheless, beside their benefits they can lead to some morbidity including EPI. In the literature, several studies have investigated EPI after bariatric surgery. Among these, Borbély et al. investigated EPI after Roux-en-Y gastric bypass (RYGB) and they found a $48 \%$ EPI frequency in distal and $19 \%$ EPI frequency in proximal RYGB patients [8]. Unlike other bariatric surgery techniques, there is no knowledge about the sleeve gastrectomy (SG)-EPI association in the literature.

\section{Aim}

In this study we assess the possible interplay between SG and EPI.

\section{Material and methods}

\section{Study design and patient population}

This is a prospective, case-control study which was conducted in the Gastroenterology and General Surgery departments of Kecioren Training and Research Hospital. Morbidly obese patients resistant to medical, diet and lifestyle therapy and therefore admitted for a sleeve gastrectomy procedure between January 2018 and April 2018 were enrolled in the study. Patients who had chronic pancreatitis, celiac disease, cystic fibrosis, diabetes mellitus, prior history of gastric and/or pancreatic surgery, prior history of acute/chronic pancreatitis and excessive alcohol consumption were excluded from the study.

Surgery procedures were done by an expert surgeon who had performed a total of nearly 1000 SG procedures. All SG procedures were done laparoscopically. Post-operative surgery related complications were not seen in any patients. Patients' medical history, gastrointestinal complaints, laboratory parameters, transabdominal ultrasonography reports and also anthropometric measurement were recorded before and after the SG procedure.

\section{Exocrine pancreatic insufficiency} definition and fecal elastase- 1 assessment

Exocrine pancreatic insufficiency was evaluated by the fecal elastase- 1 test. Fecal elastase- 1 levels $>200 \mu \mathrm{g} / \mathrm{g}$ were defined as normal, fecal elastase-1 levels $<200 \mu \mathrm{g} / \mathrm{g}$ were defined as EPI, with levels of 100 $200 \mu \mathrm{g} / \mathrm{g}$ defined as mild EPI and $<100 \mu \mathrm{g} / \mathrm{g}$ defined as severe EPI. The first fecal samples were collected a week before surgery; the second fecal samples were collected in the third month after surgery.

Fecal elastase- 1 concentrations of all samples were measured at the same time using a commercially available enzyme-linked immunosorbent assay (ELISA) kit
(BIOSERVE Diagnostics GmbH; Rostock, Germany) according to the manufacturer's instructions. All fecal samples were collected under $-20^{\circ} \mathrm{C}$ and then studied all together. Briefly, fecal samples were studied by an EL9 microplate reader and Model402 Microplate washer before fecal elastase-1 determination. Extraction buffer solution was added to fecal samples at a $10 \mathrm{mg} / 10 \mathrm{mg}$ ratio. These samples were mixed in a vortex, and after a 20 s period supernatants of samples were collected for the study. Results were given as $\mu \mathrm{g}$ elastase/g stool.

\section{Statistical analysis}

The normality of distribution of continuous variables was tested by the Shapiro-Wilk test. The Mann-Whitney $U$ test was used to compare 2 independent groups for non-normal data and the Wilcoxon test was used for non-normal data. The McNemar test was applied to investigate the relationship between categorical variable measured at 2 different time points. Statistical analysis was performed with SPSS for Windows version 24.0 and a $p<0.05$ was accepted as statistically significant.

\section{Ethical rules}

The study protocol was approved by the Institutional Ethics Committee, and the study was performed in accordance with good clinical practice and the Declaration of Helsinki. Written informed consent was obtained from each participant.

\section{Results}

A total of 40 patients were enrolled in the study. Of those, 37 patients were female and 3 patients were male. Mean age of patients was $41.9 \pm 11.25$. The baseline laboratory parameters reveal normal hepatic and renal functions as shown in Table I. Overall 8 patients had gastrointestinal complaints (bloating in 5 patients, epigastric pain in 3 patients); other individuals were asymptomatic. According to their transabdominal ultrasonography, only 6 patients had pancreatic steatosis. Esophagogastroduodenoscopy was performed routinely before surgery and gastritis was the most common endoscopic finding; neither ulcer nor erosions were seen. Prior to surgery, the mean level of body mass index (BMI) was $47.29 \pm 6.14 \mathrm{~kg} / \mathrm{m}^{2}$. Other mean anthropometric measurements were as follows: $125.35 \pm 14.17 \mathrm{~kg}$ for weight, $127.8 \pm 12.59 \mathrm{~cm}$ for waist circumference and $143.4 \pm 14.76 \mathrm{~cm}$ for hip circumference. After 3 months from surgery, all individuals were asymptomatic. Also obtained mean levels were $36.14 \pm 12.96 \mathrm{~kg} / \mathrm{m}^{2}$ for BMI, $92 \pm 12.96 \mathrm{~kg}$ for weight, $94 \pm 12.97 \mathrm{~cm}$ for waist circumference and $119.45 \pm 14.6 \mathrm{~cm}$ for hip circumference (Table I). The post-operative third month levels of weight, BMI, waist and hip circumference were 
significantly decreased compared to pre-surgery levels (Table II).

The mean pre-surgery fecal elastase- 1 level was $256.25 \pm 137.16 \mu \mathrm{g} / \mathrm{g}$ and post-surgery level was 437.7 $\pm 212.43 \mu \mathrm{g} / \mathrm{g}(p=0.001)$ (Table III, Figure 1). Prior to surgery, 20 patients had reduced fecal elastase-1 levels between 100 and $200 \mu \mathrm{g} / \mathrm{g}$. The other 20 patients had normal fecal elastase-1 levels. After surgery only

Table I. Demographic characteristics, baseline laboratory parameters and anthropometric measurements of patients

\begin{tabular}{|c|c|c|c|}
\hline Parameter & $N$ & Mean & Standard deviation \\
\hline Age & 40 & 41.90 & 11.25 \\
\hline Glucose $[\mathrm{mg} / \mathrm{dl}]$ & 40 & 92.82 & 15.27 \\
\hline $\mathrm{ALT}[\mathrm{U} / \mathrm{I}]$ & 40 & 30.95 & 21.30 \\
\hline $\mathrm{AST}[\mathrm{U} / \mathrm{I}]$ & 40 & 21.48 & 7.67 \\
\hline GGT [U/I] & 40 & 35.15 & 20.60 \\
\hline $\mathrm{ALP}[\mathrm{U} / \mathrm{I}]$ & 40 & 75.45 & 21.11 \\
\hline Amylase [U/I] & 40 & 47.88 & 15.57 \\
\hline Lipase [U/l] & 40 & 23.18 & 11.98 \\
\hline Albumin [mg/dl] & 40 & 5.075 & 4.374 \\
\hline Preoperative FE [ $\mu$ g elastase/g] & 40 & 256.24 & 137.16 \\
\hline Postoperative FE [ $\mu \mathrm{g}$ elastase/g] & 40 & 437.70 & 212.43 \\
\hline Preoperative waist circumference $[\mathrm{cm}]$ & 40 & 127.80 & 12.59 \\
\hline Preoperative height & 40 & 160.17 & 6.40 \\
\hline Preoperative weight & 40 & 120.35 & 14.17 \\
\hline Preoperative hip circumference [cm] & 40 & 143.40 & 14.76 \\
\hline Preoperative BMI & 40 & 47.298 & 6.14 \\
\hline Postoperative waist circumference $[\mathrm{cm}]$ & 40 & 94.00 & 12.97 \\
\hline Postoperative weight & 40 & 92.60 & 12.96 \\
\hline Postoperative hip circumference & 40 & 119.45 & 14.60 \\
\hline Postoperative BMI & 40 & 36.14 & 5.25 \\
\hline
\end{tabular}

FE- fecal elastase-1, BMI - body mass index.

Table II. Waist circumference, hip circumference, weight and BMI decreased significantly after surgery

\begin{tabular}{|c|c|c|c|}
\hline Variables & $\begin{array}{l}\text { Preoperative } \\
\quad(n=40)\end{array}$ & $\begin{array}{l}\text { Postoperative } \\
\quad(n=40)\end{array}$ & $P$-value \\
\hline Waist circumference & $127.8 \pm 12.59$ & $94 \pm 12.98$ & $0.001^{\star}$ \\
\hline Hip circumference & $143.4 \pm 14.77$ & $119.45 \pm 14.6$ & $0.001^{*}$ \\
\hline BMI & $47.3 \pm 6.14$ & $36.14 \pm 5.26$ & $0.001^{*}$ \\
\hline
\end{tabular}

$B M I$ - body mass index.

Table III. Fecal elastase-1 was found significantly higher after surgery

\begin{tabular}{lccc} 
Variables & $\begin{array}{c}\text { Preoperative } \\
(n=40)\end{array}$ & $\begin{array}{c}\text { Postoperative } \\
(n=40)\end{array}$ & $P$-value \\
\hline Fecal elastase-1 $[\mathrm{\mu g} / \mathrm{g}]$ & $256.25 \pm 137.16$ & $437.7 \pm 212.43$ & $0.001^{*}$
\end{tabular}

*Significant at 0.05 level; Wilcoxon test. 
4 patients had reduced fecal elastase- 1 levels between 100 and $200 \mu \mathrm{g} / \mathrm{g}$, other patients had normal fecal elastase- 1 levels. In these 4 patients, 3 patients already had reduced fecal elastase-1 levels, while only 1 patient had newly developed EPI (Table IV). Comparison of fecal elastase- 1 between pre-surgery and post-surgery revealed a significant difference $(p=0.001)$.

\section{Discussion}

Bariatric surgery is an effective treatment modality in obesity with new techniques and increased experience. Nowadays many patients are admitted to bariatric surgery clinics for morbid obesity and associated complications. Although weight loss is the primary aim of BS, surgery-associated morbidity and mortality are the leading limitations of BS procedures. Exocrine pancreatic insufficiency is one of them; prior studies demonstrate its increased frequency after RYGB [9]. However, there is no study investigating EPI after the SG procedure. Here, for the first time, we demonstrated that SG improves exocrine pancreatic functions.

Exocrine pancreatic insufficiency is a well-known complication of gastric surgery after gastric cancer. This type is a non-pancreatic cause of EPI. Various mechanisms can disrupt pancreatic secretions after gastric surgery including intestinal bacterial overgrowth due to decreased gastric acidity and altered denervation of the pancreas [10,11]. Normal pancreatic exocrine functions need optimal pH in the intestinal lumen. Increased intestinal bacterial overgrowth due to decreased gastric acidity disrupts the normal $\mathrm{pH}$ value for optimal pancreatic secretions. Also decreased cholecystokinin (CCK) secretions lead to decreased exocrine pancreatic secretions in gastrectomy patients but also this mechanism is probably responsible for EPI after RYGB [12]. Sleeve gastrectomy preserves antrum-pylorus anatomical integrity. Thus, presence of antrum and gastrin maintains gastric acidity and optimal intestinal $\mathrm{pH}$. This view can be the first explanation of our results. In addition, maintenance of anatomical integrity of the pylorus-duodenum is also an important process for normal pancreatic functions via

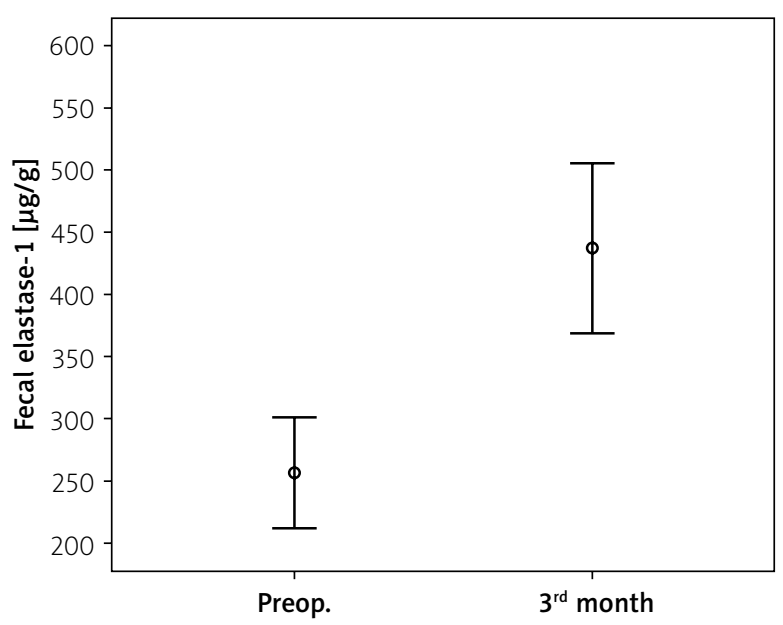

Figure 1. Fecal elastase-1 levels were significantly higher after surgery

normal CCK secretions and this is the second possible explanation of our results. In gastric bypass, digested foods are not interacted with gastric and pancreatic secretions and they pass small bowel without this interaction. Therefore exocrine pancreatic secretions via intestinal stimulation does not occur. In addition, CCK secretions diminish because of the ineffective stimulation of CCK-secreting cells. As a result, pancreatic secretions decrease and EPI occurs. In contrast with this situation, the pancreas maintains its active role in digestion after SG because of normal CCK stimulation.

Over the past two decades BS has become the most effective treatment modality for obesity. Compared to non-surgical treatment options such as medical treatments and exercise programs, weight loss and long-term protective effect from weight gain are more frequent in BS [13]. Several well-defined surgical techniques have been used in BS such as RYGB, SG, adjustable gastric banding, bilio-pancreatic diversion (BPD) and BPD with duodenal switch. Sleeve gastrectomy and RYGB are the two most commonly performed procedures. Roux-en $Y$ gastric bypass is an effective and safe procedure [14]. Prior studies showed that the long-term success of RYGB in terms of weight loss and impaired metabolic complications is nearly

Table IV. Fecal elastase was found significantly higher after surgery

\begin{tabular}{lccccc} 
Preoperative EPI frequency & \multicolumn{3}{c}{ Postoperative EPI frequency } \\
\cline { 2 - 6 } & \multicolumn{3}{c}{ Present } & Absent \\
\cline { 2 - 6 } & Count & Row N \% & Count & Row N \% \\
\hline Present & 3 & 15.0 & 17 & 85.0 \\
\hline Absent & 1 & 5.0 & 19 & 95.0
\end{tabular}

EPI - exocrine pancreatic insufficiency. *Significant at 0.05 level; Wilcoxon test. 
85\%. Also type-2 DM and hypercholesterolemia were dramatically improved after the procedure. Nevertheless, there are several metabolic-intestinal and nutritional consequences after RYGB. Absorption of iron and vitamin $D$ is affected after RYGB [15]. Exocrine pancreatic functions are also impaired after this procedure. With these metabolic-intestinal deteriorations, patients experienced several complaints associated with decreased quality of life. Sleeve gastrectomy is an effective procedure like RYGB in terms of weight loss. Compared to RYGB, nutritional deficiencies except vitamin $B_{12}$ are extremely rare. This literature knowledge highlights that SG has better nutritional-intestinal consequences compared to RYGB. Also, based on our results, we can say that the SG procedure did not adversely affect exocrine pancreatic functions but also improves pancreatic functions.

It is well known that fecal elastase- 1 is a simple, non-invasive test for the diagnosis of EPI [16]. In the medical literature, most studies used fecal elastase-1 to diagnose EPI. However, its accuracy is low despite its common use especially in severe EPI patients. There are several direct and indirect diagnostic methods for EPI but only the 13C-mixed triglyceride breath test is recommended by the FDA in clinical trials as a gold standard [17]. Recently, González-Sánchez et al. found that the diagnostic performance of fecal elastase- 1 and 13C-mixed breath test was similar in chronic pancreatitis patients in terms of EPI diagnosis [18]. Beside this recommendation and in the light of this information fecal elastase- 1 is still reliable for EPI diagnosis.

Our study has some limitations. We demonstrated early results after SG but we did not know long-term effects of the SG procedure on exocrine pancreatic functions. For this reason long-term follow-up studies are required. In our study group we did not assess pancreatic steatosis (PS) by advanced imaging techniques and this is another limitation. In obese patients it is possible to speculate that the frequency of PS may increase. Recently some studies have investigated the possible interplay between pancreatic steatosis and EPI. Kromrey et al. found that EPI was more common in patients with PS [19]. Although imaging modalities are not recommended as a diagnostic tool of EPI in current literature, investigating PS in our patients can better clarify the possible interaction between EPI and PS.

\section{Conclusions}

In our study, we found that SG relieves EPI in morbidly obese patients. Maintaining normal upper gastrointestinal system anatomical integrity is an important feature of SG and the possible explanation of our find- ings. Despite limitations of our study, this result contributes important knowledge to the literature.

\section{Acknowledgments}

We would like to thank Prof. Dr. Erkiz Oztas and Prof. Dr. Selcuk Disibeyaz for encouraging me in terms of clinical researchs associated pancreas.

\section{Conflict of interest}

The authors declare no conflict of interest.

\section{References}

1. Struyvenberg MR, Martin CR, Freedman SD. Practical guide to exocrine pancreatic insufficiency - breaking the myths. BMC Med 2017; 15: 29.

2. Keller J, Layer P. Human pancreatic exocrine response to nutrients in health and disease. Gut 2005; 54 (Suppl 6): 1-28.

3. Zsóri G, Illés D, Terzin V, et al. Exocrine pancreatic insufficiency in type 1 and type 2 diabetes mellitus: do we need to treat it? A systematic review. Pancreatology 2018; 1424 3903(18)30111-X.

4. Catarci M, Berlanda M, Grassi GB, et al. Pancreatic enzyme supplementation after gastrectomy for gastric cancer: a randomized controlled trial. Gastric Cancer 2018; 21: 542-51.

5. Neophytou H, Wangermez M, Gand E, et al. Predictive factors of endocrine and exocrine insufficiency after resection of a benign tumour of the pancreas. Ann Endocrinol 2018; 79: 53-61.

6. Friess H, Böhm J, Müller MW, et al. Maldigestion after total gastrectomy is associated with pancreatic insufficiency. Am J Gastroenterol 1996; 91: 341-7.

7. Neoptolemos JP, Ghaneh P, Andrén-Sandberg A, et al. Treatment of pancreatic exocrine insufciency after pancreatic resection. Results of a randomized, double-blind, placebo-controlled, crossover study of high vs standard dose pancreatin. Int J Pancreatol 1999; 25: 171-80.

8. Borbély Y, Plebani A, Kröll D, et al. Exocrine pancreatic insufficiency after Roux-en-Y gastric bypass. Surg Obes Relat Dis 2016; 12: 790-4.

9. Vujasinovic M, Kunst G, Breznikar B, et al. Is pancreatic exocrine insufficiency a cause of malabsorption in patients after bariatric surgery? JOP 2016; 17: 241-4.

10. Dominguez-Muñoz JE. Diagnosis and treatment of exocrine pancreatic insufficiency. Curr Opin Gastroenterol 2018; 34: 349-54.

11. Friess H, Böhm J, Müller MW, et al. Maldigestion after total gastrectomy is associated with pancreatic insufficiency. Am J Gastroenterol 1996; 91: 341-7.

12. Sabater L, Ausania F, Bakker OJ, et al. Evidence-based guidelines for the management of exocrine pancreatic insufficiency after pancreatic surgery. Ann Surg 2016; 264: 949-58.

13. Velapati SR, Shah M, Kuchkuntla AR, et al. weight regain after bariatric surgery: prevalence, etiology, and treatment. Curr Nutr Rep 2018; 7: 329-34.

14. Horsley RD, Vogels ED, McField DAP, et al. multimodal postoperative pain control is effective and reduces opioid use after laparoscopic Roux-en-y Gastric Bypass. Obes Surg 2019; 29: 394-400. 
15. Mangan A, Roux CL, Miller NG, Docherty N. Iron and vitamin D/ calcium deficiency after gastric bypass: mechanisms involved and strategies to improve oral supplement disposition. Curr Drug Metab 2018; 26: doi: 10.2174/1389200219666181026 160242.

16. Lamarca A, McCallum L, Nuttall C, et al. Somatostatin analogue-induced pancreatic exocrine insufficiency in patients with neuroendocrine tumors: results of a prospective observational study. Expert Rev Gastroenterol Hepatol 2018; 12: 723-31.

17. Domínguez-Muñoz JE, Nieto L, Vilariño $M$, et al. Development and diagnostic accuracy of a breath test for pancreatic exocrine insufficiency in chronic pancreatitis. Pancreas 2016; 45: 241-7.

18. González-Sánchez V, Amrani R, González V, et al. Diagnosis of exocrine pancreatic insufficiency in chronic pancreatitis: 13C-mixed triglyceride breath test versus fecal elastase. Pancreatology 2017; 17: 580-5.

19. Kromrey ML, Friedrich N, Hoffmann RT, et al. Pancreatic steatosis is associated with impaired exocrine pancreatic function. Invest Radiol 2019 Feb 26. doi: 10.1097

Received: 12.02 .2019

Accepted: 11.03.2019 